\title{
A Study of the Phenomenon of Spin in Airplanes.*
}

\author{
BY H. E. WIMPERIS, C.B.E.
}

$\mathrm{T}$ HE spin is a mode of motion of which we know very little. The general public are inclined to look upon it as necessarily dangerous, but this we do know it is not. It is only in rare circumstances and under fortunately rare conditions that danger arises. Nevertheless there is ample warrant for its study and for that study to be treated as one of high importance. New conditions of airplane operation are continually arising; the very increase of speed itself would ensure an entry into regions never before penetrated. Hence it is ever necessary to seek for remedies even before serious difficulty has arisen. This anticipating action always seems to me to be absolutely essential, and I am comforted by support in this view from no less an authority than Francis Bacon, who, in his essay "On Innovations", wrote: "Time is the greatest innovator; and if time in course alters things to the worse, and wisdom and counsel shall not alter them to the better, what shall be the end "?

So we have ahead of us the difficult task of diagnosing not so much actual diseases as mere symptoms, and of devising in anticipation suitable remedies. Spins from which recovery is difficult may be rare, but since from time to time they are reported, a vigorous investigation becomes necessary. Though that investigation is far from having been completed -indeed in some ways it is little more than begunthe interest taken by everyone in flying makes some account of our present efforts worth attempting, whilst the very complexity of the phenomenon is in itself a challenge.

\section{The SPIN.}

The first step will be to describe what constitutes a spin, why certain forms of spin present difficulty, why the obvious remedies fail, and the form which the studies now in hand have taken.

Almost all airplanes are built nowadays to be stable in flight; if any small disturbance to their normal attitude occurs the craft tends by its own virtue to return to its previous attitude. The naval architect has always aimed so to design ships that they have this great quality. Indeed, a ship the stability of which depended upon the clever balancing action of its steersman would be decidedly unpopular.

Now how does an airplane achieve stability? If one suspends a model airplane from a point above its centre of gravity it hangs in stable equilibrium. If slightly displaced it returns. When gliding in flight the upward pull of the string has to be replaced by the resultant of all the upward air forces on the wings and tail plane. If stability is to be obtained, this vertical resultant must pass through the centre of gravity of the craft. The usual way of ensuring this is to give an upward tilt to each wing (the dihedral angle), so that the air forces on the two sides are inclined and meet at a point in the vertical plane of symmetry. The relative inclina-

* Discourse delivered at the Royal Institution on Friday, May 2.

No. 3169, Vor. 126] tions of wing and tail plane can be adjusted in just the same way, and thus the resultant of all the vertical air forces is made to pass through the centre of gravity so that the airplane behaves as though suspended at an imaginary point above the airplane.

This is the condition of ordinary straight flight: centrifugal force does not come into the picture. With circular motion, however, it does. The airplane banks as it turns, the air forces bank with it, and their resultant force balances gravity by its vertical component and the centrifugal force-due to the turn by its horizontal one. Hence motion in circles can be just as steady and just as normal as in straight flight. Inconvenient and even dangerous as the actual spin may sometimes be, the spinning airplane is not, so to speal, conscious of doing anything wrong. Its behaviour is purely normal and quite virtuous. There is nothing in it to suggest the vicious circle. When the radius of the turn is made gradually less and less, the angle of bank grows greater and greater until the vertical component of the air forces can no longer balance gravity and the nose drops; the motion then becomes a kind of tight corkserew. The motion is still steady and is quite safe. It is safe because the pilot can convert the motion into ordinary straight level flight whenever he wishes to do so. If, however, the corkscrew is made tighter and tighter the motion of the airplane may suddenly change to one in which the air forces on the machine will automatically tend to make it tighter still. This is the spin.

Before the essential features of the phenomenon of spin can be grasped, it is necessary to consider in slightly more detail the nature of the forces which act on the wings of an airplane.

\section{Autorotation.}

Airplane wings are of various shapes--some have concave undersurfaces and some convex-but all alike when inclined at an angle to the wind (the angle of incidence) give rise to an upward lift force and a small rearward drag. It is important to notice the manner in which the lift force changes as the angle of incidence increases. To begin with, the lift force doubles for each doubling of this angle, but once the latter has reached a certain amount, the lift force increases less rapidly until at a particular angle (known as the angle of stall and usually about $20^{\circ}$ ) the lift force ceases to increase at all, and instead falls off rapidly as the angle is still further increased. When this happens there is produced a new phenomenon altogether. For if any sudden "bump in the atmosphere causes the right wing (let us say) to drop and therefore to meet the air at a steeper angle, the lift force on that wing will ipso facto become less and the wing will tend to drop still further. In fact, the whole wing will tend to go on turning. This is the phenomenon of autorotation. It can easily be exhibited in a wind 
tunnel by so mounting an aerofoil about a suitable axis as to give it a large angle of incidence; once the air stream is turned on the aerofoil will be found to be quite ready to rotate in either direction once some external cause has given it an initial push in that direction. (An experimental demonstration of autorotation was given at this point.)

The speed of autorotation depends upon the aerofoil section and the angle of incidence. In the case of a complete airplane it depends also on the disposition of the wings particularly in relation to each other and upon the arrangement of parts in the tail of the machine. It is the existence of the many factors which govern the occurrence and speed of autorotation which makes the study of the spin so complicated, that unless the problem is reducible to a few bare essentials a purely theoretical solution is scarcely to be anticipated.

When describing the tight corkscrew as a mode of motion I mentioned that this motion might suddenly change to the spin. This will happen if the angle of incidence should rise to the value at which for that particular wing section, and aerodynamic arrangement, autorotation sets in. Thereupon the forces acting on the airplane speed up the rotation and tend generally to take charge. Sometimes the machine is said to be 'locked into' the spin, but this language though understandable is really over strong, since the pilot by putting down the nose of the craft can bring the angle of incidence below that of autorotation; at once the autorotation couple dies away and the spin ceases.

How is a true mental picture of this complicated motion to be made? There are the resultant air force the centrifugal force, and the gravitational attraction all acting on the airplane. We know that the last-named always acts vertically downwards, whilst the centrifugal force acts at right angles to the axis of spin. For equilibrium these forces must be balanced by the air forces, hence the resultant of these latter must have a vertical component equal to the weight, a horizontal and radial component equal to the centrifugal force, and a horizontal and tangential component of zero. This last has to be zero, since in steady motion there can be no acceleration in that direction, and any force necessary to drag the tail round must be supplied by a suitable component of the air forces which act on the aerodynamic surfaces. The resulting motions are known, but no disentanglement of the various air forces due to lift, drag, and sideslip has yet been made.

It was Bairstow who first suggested that the phenomenon of spinning could be imitated in a wind channel by setting an aerofoil at such an angle that self-rotation must occur. This is made clear in an Aeronautical Research Committee report dated October 1918. In this report Relf and Lavender showed not only why autorotation took place, but also how to calculate the rate of spin. Model tests showed excellent agreement with their prediction. The rate of spin was found to increase with the angle of incidence, and in a chosen example the rotational speed rose from about $1 \frac{1}{2}$ spins per second to 2 spins per second when the incidence was increased from $20^{\circ}$ to $30^{\circ}$.

It is clear from what has already been said, that a lift curve which did not drop after the stalling point would be much less likely to lead to autorotation, or would in any case delay it to so large an angle of incidence that it would be very rare for an airplane to be in the attitude at which spinning could occur. The so-called 'flat-topped lift curve" has this virtue in varying measure, as also have airplanes with slotted wings, for then the stalling angle is very large and could usually only be reached, if at all, by a deliberate jerk to the whole machine. Getting into a spin would then be a piece of voluntary acrobatics and not a pitfall awaiting the unwary. 'Pitfall' may seem a strong word, especially as the phrase of 'locking into' a spin has been deprecated on the ground that, after all, the pilot holds the key of the motion in the right use of his control levers. It seems, however, that certain forms of airplane have so high an autorotation couple that, given time to attain the full corresponding rate of spin, the centrifugal forces due to the unsymmetrical distribution of mass in the airplane may oppose and overcome the control force exerted by the pilot's actions, and this may happen the more readily on account of the spin having become so flat (the airplane being less in clined to the horizontal plane than the vertical) that the control organs in the tail, in the fin, and rudder, are shielded from effective action by the tail plane. At the same time the physiological action of the rotational forces on the pilot may hinder his actions. If one casts a kind of airstream 'shadow' at $45^{\circ}$ behind the tail plane, it will be seen that but little of the fin and rudder area can be effective in slowing up the undesired rotation or indeed in affecting it in any way.

The most elementary way of preventing this rapid spin from developing is to increase the size of the vertical tail surfaces, the fin and rudder, and to lengthen the fuselage so that a greater leverage is available. This leads to an increase of weight in the tail, and puts the centre of that weight still farther aft-both these actions lead to an increase in what are called the inertia couples due to the centrifugal force. The effect of these inertia couples is, as I will describe in a moment, to oppose the pilot's control of the machine, and so neutralise wholly or partly the good effect, from another point of view, of the larger tail surfaces and greater leverage. Nevertheless, there is sometimes an advantage on balance to be gained in this way, and for some time a ready criterion of spinning tendencies was used in America which depended principally upon whether or not the tail organs were inside a semicircle based on the wing span.

\section{INERTIA COUPLES.}

The effect of an inertia couple can be illustrated by taking the simple case of a rod having equal heavy masses at each end and pivoted at its centre of gravity. If one holds such a system at arm's length and turns round rapidly the rod takes up a horizontal position. This happens because the

No. 3169 , Vor 126] 
centrifugal force on each mass is proportional to its radius of rotation, hence the mass which starts farthest out tends to go still farther out, and stability is only reached when the rod is at right angles to the axis of rotation. If a second similar rod were fastened at right angles, the forces upon it would balance those on the first rod. The combination would be neutral and would take up a neutral position. Hence an airplane with its masses symmetrically disposed would be free from such couples and free therefore from the peculiar difficulties of a flat spin. In practice, however, airplanes cannot be so made, and as a fair example of what occurs in practice the case of the wellknown Bristol Fighter airplane may be cited. Here the moments of inertia about the longitudinal axis and the transverse axis are found to be about equal, whilst that about a vertical axis is half as much again.

A simple calculation shows that for any given rate of rotation this disturbing couple reaches a maximum when the fore and aft line of the airplane is inclined downwards at $45^{\circ}$. Once that angle is exceeded the couple grows less. When, therefore, an airplane is being brought out of a very flat spin the pilot has to exert a control not merely large enough to balance the inertia couple at the moment, but also large enough to overbalance this growing couple as the barrier angle is reached. The barrier angle will come at exactly $45^{\circ}$ if the rate of spin remains constant, if it does not it may be somewhat above or below this angle. But a barrier there will in general always be, and recovery from any flat spin must depend on its being satisfactorily surmounted. (An experimental demonstration was given to show the effect of the inertia couple in raising the nose of the fuselage.)

A typical pilot's impression of the change from the ordinary spin to the flat spin appeared in one report as follows: "After the first two or three turns, which were relatively steep, the nose came up and the machine settled down to an exceptionally steady spin at moderately large incidence and a quick rate of rotation. There was no noticeable jerkiness during the turn."

(To be continued.)

\section{The Bristol Meeting of the British Association.}

\section{Programmes of Sections.}

\section{Mathematical and Physical Sciences.}

$\mathrm{T}$ HE presidential address of Section A (Mathematical and Physical Sciences) will be delivered on Monday, Sept. 8, by Dr. F. E. Smith, who has chosen as his subject "The Theories of Terrestrial Magnetism." On each of the other days a considerable portion of the available time will be taken up by a series of related papers. Thus on Thursday, Sept. 4, there will be a discussion on "The Meteorological Relations of Atmospherics," in which Dr. R. A. Watson Watt, Prof. E. V. Appleton, M. R. Bureau, Dr. F. Schindlehauer, and Mr. M. A. Giblett will participate. On the following day there will be a series of papers dealing with aspects of the solid state, the contributors being Prof. J. E. Lennard-Jones, Prof. W. L. Bragg, Dr. J. D. Bernal, and Dr. F. Bloch. These will be followed by a brief discussion, opened by Prof. Heisenberg. On Tuesday, Sept. 9, there will be a discussion on flow in gases, and the aerodynamical and meteorological aspects of this subject will be dealt with by Mr. E. Ower, Mr. F. C. Johannsen, Mr. G. Bilham, and Mr. M. A. Giblett. The programme contains also the usual papers on particular investigations.

Many distinguished foreign visitors are contributing to the proceedings. In addition to those mentioned above, Prof. M. Siegbahn will present a paper on "The Highly Ionised Spectra in the Extreme Ultra-Violet," and the section will probably listen to other eminent visitors.

There will be a strong Sub-Section of Mathematics, and twenty papers appear on its programme. Several of these will be of interest to physicists and others. Thus Prof. S. Brodetsky will read a paper on "The Einstein Field-Theory," and Prof. A. C.
Dixon will discuss integral equations, a subject which is rapidly becoming important in physical investigations. There will also be a paper on modern Babbage calculating machines by Dr. L. J. Comrie.

\section{Chemistry.}

For his presidential address to Section B (Chemistry) Prof. G. T. Morgan, director of chemical research in the Department of Scientific and Industrial Research, has chosen the title "A State Experiment in Chemical Research". $\mathrm{He}$ will discuss the development of the Chemical Research Laboratory at Teddington since its beginning five years ago. His account of the investigations now in progress at that institution will be illustrated by an exhibit of preparations, apparatus, and models of chemical plant.

On Friday, Sept. 5, a discussion will be held on the present position of the British dyestuff industry -a topic which is particularly opportune in view of the impending lapse of the Dyestuffs (Import Regulations) Act in January 1931. In opening the discussion, Prof. A. G. Green will review the development of the British industrysince 1901; when he gave an account of the position at the Glasgow meeting of the Association. Subsequent speakers will be Prof. J. F. Thorpe, president of the Chemical Society and a member of the Dyestuffs Industry Development Committee, Sir William Pope, and the following members of the Dyestuffs Industry Development Committee: Messrs. C. J. T. Cronshaw, G. Holden, J. Morton, and Major L. B. Holliday. Mr. W. J. U. Wooleock will sum up the debate.

Another important discussion will be opened on Monday, Sept. 8, by Dr. F. L. Pyman, on "Chemo-

$$
\text { No. 3169, VoL. 126] }
$$

